\title{
Automated System for the Analysis of Heart Monophasic Action Potentials
}

\author{
DG Tsalikakis $^{1,2}$, DI Fotiadis ${ }^{1,2,3}$, T Koletis ${ }^{3,4}$, LK Michalis L $^{3,4}$ \\ ${ }^{1}$ Unit of Medical Technology and Intelligent Information Systems, Dept. of Computer Science, \\ University of Ioannina, GR 45110 Ioannina, Greece \\ ${ }^{2}$ Biomedical Research Institute-FORTH, GR 45110 Ioannina, Greece \\ ${ }^{3}$ Michaelidion Cardiology Center, GR 45110 Ioannina, Greece \\ ${ }^{4}$ Division of Cardiology, Medical School, University of Ioannina, GR 45110 Ioannina, Greece
}

\begin{abstract}
In this paper we present an automated system, which can be used in real time for the analysis of heart monophasic action potentials (MAPs) in order to analyze the behaviour of the myocardium under pathological or normal conditions. The system can be described as a three-stage procedure. In the first stage MAPs are acquired using epicardiac electrodes or MAP Franz catheters and the noise is removed using a combination of notch and band pass filters. In the second stage several features of the signal are extracted. In the third stage statistical analysis is performed. The obtained results can be used for the assessment of the electrophysiological status of the atrial muscle, ventricular muscle, and the evaluation of the effects of antiarrhythmic drugs. The validation of the system indicated that it can provide with reliable information on the myocardium condition.
\end{abstract}

\section{Introduction}

Monophasic action potentials (MAPs) are exrtacellular recordings that have been found to correlate sufficiently with intracellular action potentials (IAPs) [1]. There are many reports investigating the effects of electrophysiological and pharmacological interventions in the MAP of the intact heart [2,3]. The development of contact MAP electrodes allows quick and easy recording of MAPs in clinical settings [2,3]. Standard recording techniques using paper traces often lack quality and resolution, and require time consuming manual processing. There have been several attempts to automate the analysis of sampled MAPs $[4,5,6]$ but only few of them have been evaluated for their accuracy and reliability [4].

It is the aim of our work to develop an automated system for the extraction and analysis of MAP features to understand myocardium condition. The system consists of three stages: signal acquisition, feature extraction and statistical processing. The MAP features which are extracted with high accuracy are: MAP amplitude, action potential duration (APD) at all repolarization levels (1090\%), the baseline and amplitude. The proposed system has been used for the analysis of MAPs from two series of experiments.

The system performs well despite the morphological differences of the signals. The drug effect on MAPs is recorded and useful input can de acquired for the myocardium condition.

\section{Materials and methods}

The proposed MAP automated analysis system consists of three stages: (a) signal acquisition, (b) feature extraction, and statistical feature processing. Those stages are described in detail below.

\subsection{Signal acquisition}

The acquisition module includes the epicardiac probe or the MAP Franz catheter, the MAP preamplifier, the A/D converter, and software, which has been developed using Labview 6.1. Parameters such as sampling rate, recording time and type of filter can be adjusted to achieve the desirable MAP signal quality criteria as stable baseline, MAP morphology, fast upstroke, upstroke duration $<2 \mathrm{~ms}$ and amplitude [5]. The recording software has been validated with a pulse generator, recording and analyzing a sequence of 400 square waves with amplitude $1 \mathrm{mV}$ and cycle length 400, 300, 200, 150 msec.

The MAP signal is heavily contaminated by noise (power line interference and motion artifacts), which is removed by a combination of filters (notch, low, band and high pass). Due to the power line interference at 50 $\mathrm{Hz}$ the digital notch filter has a transfer function 
$H(z)=\frac{1-2 \cos \left(\omega_{0}\right) z^{-1}+z^{-2}}{1-2 r \cos \left(\omega_{0}\right) z^{-1}+r^{2} z^{-2}}$ where $\omega_{0} \quad$ is the angular frequency corresponding to the interference frequency and $Q=\frac{\omega_{0}}{2(1-r)}$ is a quality factor with $r<1$ [13].

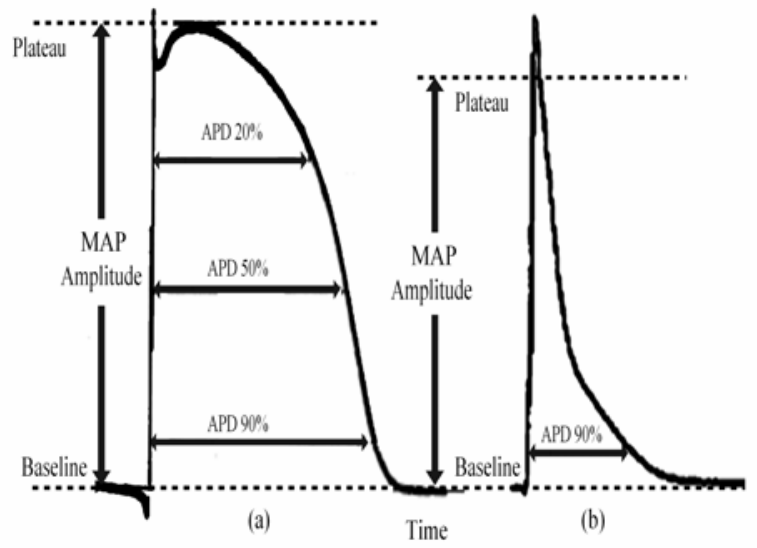

Fig. 1. Morphological differences and MAP characteristics in: (a) pig and (b) rat.

\subsection{Feature extraction}

In this stage the MAP signal features are extracted. The MAP morphology and the MAP used features for pigs and rats are shown in Fig. 1. The feature extraction procedure is illustrated in Fig. 2. Initially, the used threshold values are set and the signal is fed into the system. The algorithm can extract upstroke, the baseline, the plateau and the APDs. To make easier the analysis a window is used, whose length is adapted when an upstroke is found. A baseline is acceptable when it compares well (using a threshold value) with the average of the previous five baselines. The feature extraction ends when both plateau and APDs are detected. The algorithm starts again looking for a new upstroke starting from the end point of the last MAP. The whole procedure is based on the following rules:

- The start time of the MAP is determined being the upstroke of the detected MAP.

- For 5msec sliding window in the range (upstroke-30msec) to (upstroke-10msec) the average value of the first derivative is computed. The window with the lowest average derivative is selected and the baseline is defined as the average voltage of this window.
- $\quad$ Similarly, for $5 \mathrm{msec}$ sliding window in the range (upstroke+10msec) to (upstroke+30msec) the average value of the first derivative is computed. The window with the highest average derivative is selected and the average voltage of this window defines the MAP plateau.

- The amplitude of the MAP is derived as the absolute difference between the plateau and the baseline.

- If the percentage of repolarization is defined as $x$ the corresponding voltage of the MAP signal from the baseline is: $\mathrm{V}_{\mathrm{X}}=$ Plateau $-\frac{\mathrm{x}}{100}$ Amplitude (in $\mathrm{mV}$ ).

- The APDx\% is given as the difference between the ending point of baseline and the projection of $\mathrm{V}_{\mathrm{X}}$ in the time axis.

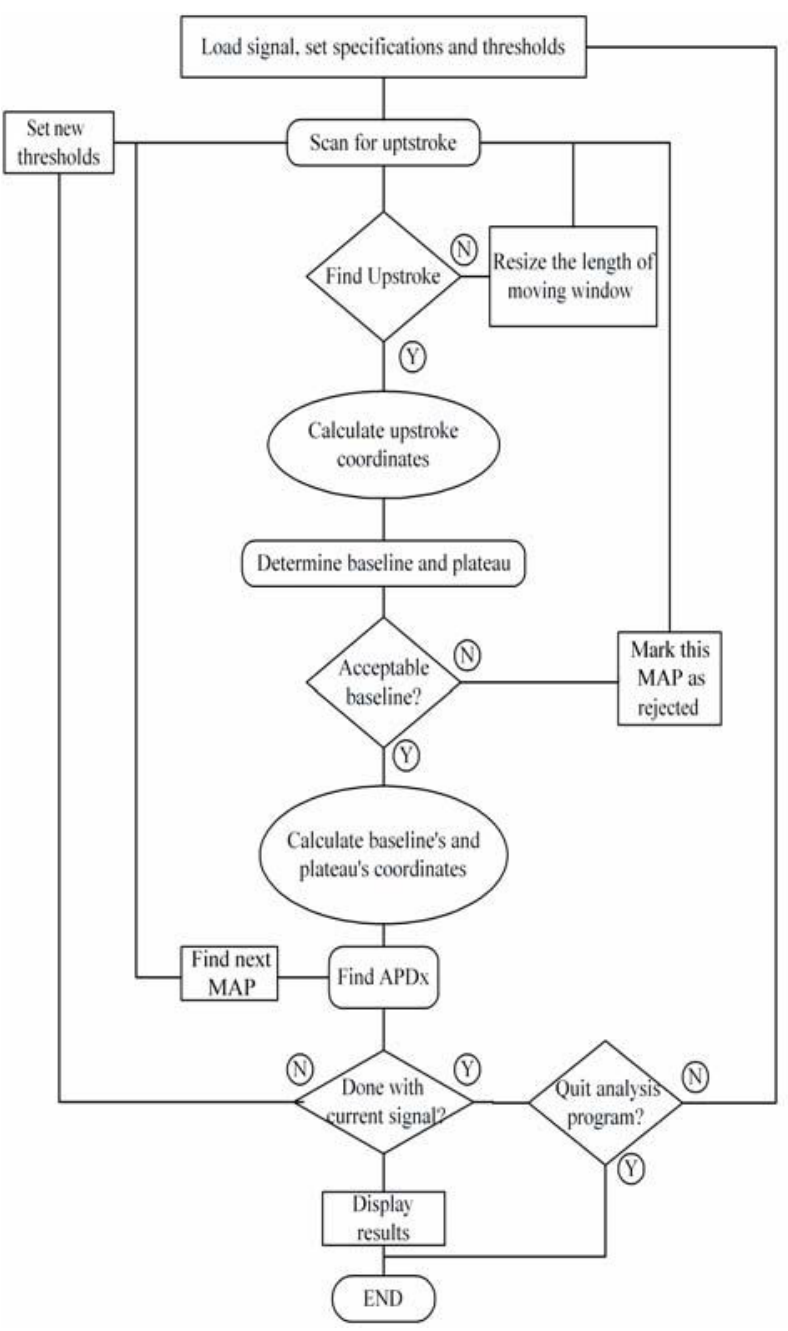

Fig. 2 . Feature extraction procedure. 


\subsection{Statistical feature analysis}

The average value and standard deviation of upstroke intervals, baseline intervals, plateau intervals and APDs are computed. Those must be extracted for various conditions, even for cases when antiarrhythmic drugs are used. Those values correspond to certain myocardium condition profiles and easily can be compared with existing data from the literature.

\section{Experiments}

We performed two series of experiments. In the first the use of growth hormone as an antiarrhythmic agent, in arrhythmias caused from coronary artery blockage is investigated. We recorded MAPs from 10 pigs to assess local ischemic electrophysiological changes. The recordings were performed endocardiacly in left ventricular. The effect of BQ-102 ET-A (endothelin receptor antagonist) after the myocardium infraction was examined in the second series of experiments. Myocardium infraction experimental simulated with the blockage of artery lad. Experiments in 20 rats were performed and we recorded epicardiac MAPs, 9 of those were in normal condition and 11 suffered from left ventricular myocardium infraction and received BQ-102 ET-A treatment.

\section{Results}

We analyzed about 2000 MAPs from both series of experiments. All MAP signals were automatically analyzed and an example of the calculated rat MAP features is shown in Fig. 3. In this example the sampling frequency was $1 \mathrm{kHz}$ and no filters were used. In Fig. 4 baseline intervals with APD75\% are shown.

\section{Discussion and conclusions}

We have developed an automated system for the analysis of MAPs. The system includes hardware and software modules and operates in real time. The obtained values of the necessary for diagnosis signal features follow the proposed quality criteria. This is due to efficient removal of noise, which appears to be a critical factor, especially during epicardiac recordings. The system has been tested in obtained MAPs from two diverse series of experiments. The system responds well to a wide variety of application in clinical electrophysiology as drug treatment and local acquisition of MAP signals.
The system can be further expanded to include medical knowledge in order to provide with diagnosis options.

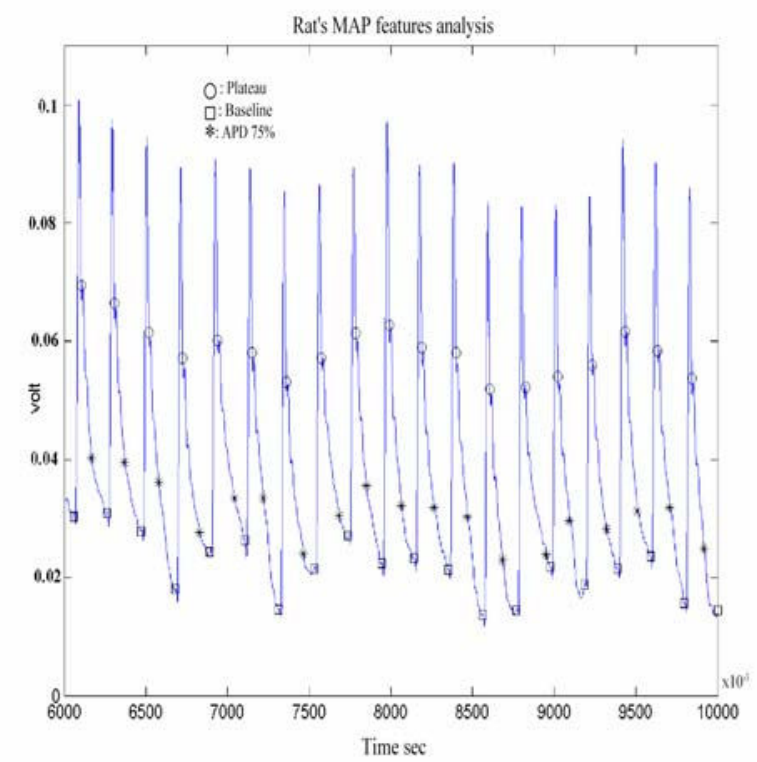

Fig. 3. Rat's MAP signal analysis for a $4 \mathrm{sec}$ interval.

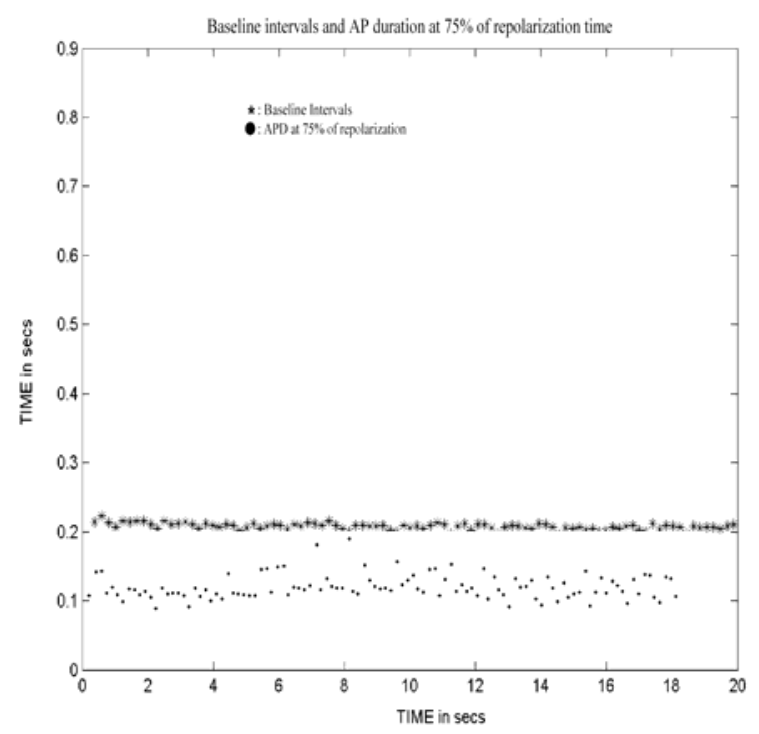

Fig. 4. Graphical display of baseline intervals and APD $75 \%$ for a $20 \mathrm{sec}$ interval 


\section{Acknowledgements}

This work is part funded by the Greek Secretariat for Research and Technology ( P.E.N.E.D: "Investigation of growth hormone in myocardium after infraction" 01ED511).

\section{References}

[1] Franz MR. Current status of monophasic action potential recording: theories, measurements and interpretations. Cardiovascular Research 1999;41:25-40.

[2] Xie JT, January CT. The monophasic action potential. technique and its application in cardiac electropharmacology. Meth Find Exp Clin Pharmacol 1993;15:557-567.

[3] Franz MR. Monophasic action potential: Bridging cell and bedside. Futura Publishing, New York 2000.

[4] Franz MR, Kirchhof PF, Fabritz CL, Zabel M. Computer analysis of monophasic action potential: Manual validation and clinically pertinent applications. PACE 1995; 18:16661678.

[5] Dickenson DR, Davis DR, Beatch GN. Development and evaluation of a fully automated monophasic action potential analysis program. Med.Biol.Eng.Comput 1997;35:653-660.

[6] Knollmann BC, Katchman AN, Franz Mr. Monophasic action potential recordings from intact mouse heart: Validation, regional heterogeneity and relation to refractoriness. J Cardiovasc Electrophysiol 2001;12:12861294.

[7] Fabritz L, Kirchhof P, Franz MR. Prolonged action potential durations increased dispersion of repolarization, and polymorphic ventricular tachycardia in a mouse model of proarrhythmia. Basic Res Cardiol 2003;98:25-32.

[8] Dean JW, Lab MJ. Effects of changes in load of monophasic action potential and segment length of pig heart in situ. Cardiovascular Res 1989;23:887-896.

[9] Behrens S, Li C, Knollmann BC. Dispersion of ventricular repolarization in the voltage domain. PACE 1998;21:100107.

[10] Merkely B, Vago H, Geller L. Effects of radiofrequency ablation on monophasic action potential. IEEE Eng in Med \& Biol 2002;69-73.

[11] Zabel M, Lichtlen PR, Haverich A, Franz MR. Comparison of ECG variables of dispersion of ventricular repolarization with direct myocardial repolarization measurements in human heart. J Card Elecrt 1989;9:12791284.

[12] Danik S, Cabo C, Chiello C. Correlation or repolarization of ventricular monophasic action potential with ECG in murine heart. Am J Physiol Heart Circ Physiol 2002;283:H372-H381.

[13] Christov II, Daskalov IK. Filtering of electromyogram artifacts from the electrocardiogram. Med Eng \& Physics 1999;21:731-736.

[14] Daskalov IK, Christov II. Electrocardiogram signal preprocessing for automatic detection of QRS boundaries. Med Eng \& Physics 1999;21:37-44.

[15] Sahakian AV, Peterson ML, Shkurovich, Hamer M, Votapka T, Ji T, Swiryn S. A simultaneous multichannel monophasic action potential electrode array for in vivo epicardial repolarization mapping. Trans on Biomedical Engineering 2001;3:345-352.

[16] National Instruments, Labview data acquisitions basics manual. National Instruments, Austin, TX 1998.

[17] Stambler BS, Wood MA, Ellenbogen KA. Pharmacologic alterations in human type I atrial flutter cycle length and monophasic action potential. American collefe of cardiology 1996;2:453-461.

[18] Witkowski FX, Penkoske PA. Proceedings, IEEE Eng in Med \& Biol 1990;12:595:596

[19] Hondeghem LM, Carlsson L, Duker G. Instability and triangulation of the action potential predict serious proarrythmia, but action potential duration prolongation is antiarrhythmic. Circulation 2001;103:2004-2013.

[20] Action potential characteristics and arrhythmogenic properties of the cardiac conduction system of the murine heart. Circ. Res. 2001;89:329-335.

Address for correspondence.

Dimitrios Fotiadis.

Unit of Medical Technology and Intelligent Information

Systems, Dept. of Computer Science, University of Ioannina, P.O box 1186, GR 45110, Ioannina, Greece.

E-mail: fotiadis@cs.uoi.gr 